\title{
Effects of an assisted living platform amongst frail older adults and their caregivers: 6 months vs. 9 months follow-up across a pilot field study
}

\author{
Lucile Dupuy $\mathrm{PhD}^{\mathrm{a}, \mathrm{b}, *}$ \\ Hélène Sauzéon $\mathrm{PhD}^{\mathrm{b}, \mathrm{c}}$
}

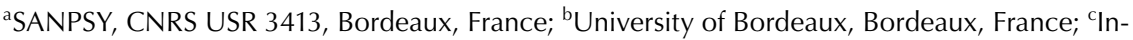
ria Bordeaux Sud-Ouest, Talence, France; *Corresponding author: lucile.dupuy@u-bordeaux.fr

L. Dupuy, H. Sauzéon. Effects of an assisted living platform amongst frail older adults and their caregivers: 6 months vs. 9 months follow-up across a pilot field study. Gerontechnology 2020;19(1):16-27; https://doi.org/10.4017/gt.2020.19.1.003.00 Background Ambient Assistive Living (AAL) technologies provide promising solutions to support aging-in-place but still lack ecological validation. Frail older Individuals (FIs) are at high risk of losing their autonomy but are also acknowledged to be an optimal target population for dependency prevention based on environmental supports. In this context, a recent study revealed some promising short-term benefits of an AAL platform in supporting Fls' everyday functioning and releasing caregiver burden. Research aim Complementarily to this first study, we aimed to address near and far effects of an AAL platform on Fls' everyday functioning and caregiver burden. Method HomeAssist is an AAL platform providing environmental support thanks to services including activity verification, safety alerts, reminders, communication and leisure activities. HomeAssist efficacy has been evaluated in a field study including a nine-month deployment in the home of sixteen cognitively healthy (MMSE > 25) Fls living alone. Several efficacy measures were taken and compared to a control group $(n=16)$, matched by age, gender, cognitive functioning and frailty condition. These measures included Fls' autonomy (self-reported and reported by a caregiver) and caregiver burden (for supporting Fls' autonomy and professional burnout) and were assessed at baseline, six, and nine months later. Results Our results indicated that: (1) Fls' autonomy reported by caregivers is improved over time for the equipped group, with a greater extent after 9 months; (2) Fls' self-perception of everyday functioning remained constant after 9 months for the equipped group, whereas it slightly decreased for the control group; and (3) caregivers reported less burden across time in the equipped group compared to control group; while HomeAssist intervention did not significantly impact professional burden. Conclusion Taken together, this suggests that near effects of HomeAssist are not only due to novelty, but rather benefits from HomeAssist intervention.
\end{abstract}

Keywords: Ambient assisted living, aging in place, caregiver burden, everyday functioning

\section{INTRODUCTION}

Ambient Assisted Living (AAL) technologies, referring to assistive technologies that are sensitive, adaptive and responsive, to their users and their environment (Rashidi \& Mihailidis, 2013), are now involved in every part of our life: communication, activities of daily living, healthcare, safety of the person and the house (Blackman et al., 2015). These AAL can improve everyone's life and have a promising future to support older adults' autonomy, and particularly frail individuals (FI) (Dupuy, Froger, Consel,.Sauzéon, 2017). Although older adults are open and willing to use technologies (e.g., Berkowsky et al., 2018), the demonstration of technology efficacy to support Fls' autonomy has yet to be reinforced, notably in terms of durability effects. Addition- ally, AAL technologies should be able to relieve caregivers from caregiving burden, as caregivers play a major role in older adults' autonomy and technology adoption (e.g., Peek et al., 2014), but this facet is often omitted in the evaluation of AAL efficacy (Liu et al. 2016). Consequently, the present pilot field study aimed to address near and far effects of an AAL platform on Fls' everyday functioning and caregiver burden.

After a brief definition of frailty occurring with age, an overview of current and emerging AAL technologies is presented with a focus on those supporting older adults' independent living. Current research challenges related to the assessment of AAL benefits are then highlighted to move forward more evidence of the AAL efficacy to improve frail 
older adults and caregivers' everyday life.

\section{Frailty syndrome in aging and caregiver burden} With the aging of the population, frailty syndrome has acquired an increased interest, as its prevalence among seniors is becoming significant. Indeed, a 2012-study found that $10 \%$ of the population aged 65 and over, and $26 \%$ over 85 years old was experiencing frailty, and this proportion is expected to grow in the following years (Collard et al., 2012).

Not to be a disease or pathological condition, frailty is a clinical syndrome and can be defined as an increased vulnerability due to a poor ability to deal with age-related decline in physical, cognitive and physiological conditions (Clegg et al., 2013). Fried and colleagues seminal work defined frail individuals (FIs) as individuals meeting three or more of these five criteria: weak strength, slow gait speed, self-reported exhaustion, unintentional weight loss, and low physical activity (Fried et al., 2001). This state of vulnerability can lead to several negative outcomes in everyday life, including falls, hospitalization, autonomy loss, and death (Fried et al., 2001; Avila-Funes et al., 2008). Another consequence of frailty is the increased need for caregiving, which may result in a burden for family and professional caregivers (McFall \& Miller, 1992). Burden possesses various definitions and authors separate the subjective burden, corresponding to the feelings, attitudes, and emotions elicited by the caregiving tasks; and the objective burden, referring to the caregiving events, happenings and activities (Montgomery, Gonyea \& Hooyman, 1985).

Regarding interventions to support frailty, some studies highlighted that FIs are an optimal target for non-pharmaceutical intervention (Morley et al., 2014). Therefore, several environmental interventions have shown their potential to support FIs, including exercising (Theou et al., 2011), or changes in food regimen (Morley et al., 2014). Other possible environmental supports come from assistive devices, having the potential to either adapt the environment to make it more accommodating, or to provide compensatory supports for a capability loss thanks to environmental prompts for initiating and/or helping the task realizing. (Morrow \& Rogers, 2008). Environmental interventions can be effective for helping Fls to perform everyday activities, or even to reduce their functional degradation. The spectrum of benefit expectations from environmental interventions is large, ranging from performance renormalizing, improved everyday activities, to preventing their deterioration or slowing down their degradation. Furthermore, these environmental supports could alleviate caregivers' role, hence reducing their burden.

\section{AAL technologies for older adults}

In the past few years, research and industry have provided a great deal of effort into the development of AAL technologies to support older adults' autonomy and well-being in various aspects of everyday life, including activities of daily living (ADL), communication, and safety (e.g., Blackman et al., 2015; Rashidi \& Mihailidis, 2013).

Examples of AAL technologies providing support for ADL include CASAS (Rashidi \& Cook, 2009), COACH (Mihailidis et al., 2008), or Lee and Dey's pill reminder (Lee \& Dey, 2014), monitoring everyday activities such as toileting, meal preparation or medication taking; and dispensing prompts or reminders. Regarding communication, we can cite the PRISM system (Czaja et al., 2017), InTouch (Neves et al., 2015) or Wayve (Lindley, 2012), providing a sense of connectedness by sharing pictures and messages with family and friends. Examples of AAL for safety purposes include iFall (Sposaro \& Tyson, 2009) and cAALyx (Rocha et al., 2013), tracking vital signs or falls, with the possibility to contact caregivers or emergency services in case of an unusual situation. Therefore, AAL has the potential to integrate a range of technologies, products, and services for promoting aging in place.

Moreover, it is noteworthy that caregivers are also often involved in AAL-based intervention. Therefore, when evaluating the efficacy of $A A L$ to support older adults' autonomy, the care receiver-caregiver dyad should deserve more consideration by including secondary outcome measures related to caregivers' burden.

\section{Evaluating the benefits of AAL technologies for older adults and their caregivers}

Despite the increasing amount of "specifically and appropriately designed" technologies for older adults, evidence of their efficacy is still expected. Even when an experimental validation is performed, it often does not meet rigorous experimental standards, i.e. a long enough evaluation process, a significant sample size, a control condition, validated tool measurements, and an ecological situation (Slim et al., 2003). For example, Queiros et al. (2015) performed a systematic review of the AAL literature, analyzing a total of 1,048 studies. They reported that only $10 \%$ of these studies were related to user issues (accessibility and usability, in particular), clearly revealing the technology-oriented approach of the AAL field for older adults. Also, among the $13 \%$ of technologies with a practical purpose, only $0.04 \%$ ( 6 studies) have been tested in field trials. As already observed in a previous review (Reeder et al., 2013), these field studies are reported as lacking empirical evidence of AAL efficacy, mostly due to the study design with often 
small sample sizes, non-standardized measures (i.e., self-made measures), experimental home setting (rather than real homes), and an absence of a control group. Similarly, and more recently, Liu et al., (2016) found that only a third of their reviewed papers performed an experimental validation, and ecological validation studies in the home of participants were very rare.

Among existing ecological validation studies for AAL benefits toward older adults, we can cite the recent randomized controlled trial carried out in the PRISM trial (Czaja et al., 2017). In their field study, 300 older adults benefitted at home either from a mini-computer with communication and leisure services (the Personal Reminder Information and Social Management - PRISM system), or a notebook with similar content, over an 18-month period. Based on standardized measurements, their results showed a significant reduction of social isolation and loneliness in both groups, with a greater improvement for the PRISM condition.

Regarding field studies involving AAL with sensors, we can cite the recent work of Rantz and colleagues (2017). In this randomized and controlled study, 86 older adults living in assisted living communities received a suite of environmental sensors (motion sensors, mattress bed sensors, and gait sensors) in order to measure their mobility and detect falls. This group of users was compared to 89 older adults receiving standard care. Results showed that one year later the intervention group declined more slowly than the control group in terms of mobility and that in case of functional decline, nurses could detect and intervene earlier with the intervention group compared to the control group. A less recent study presenting good methodological standards is the one of Tomita et al. (2007). Indeed, in their study, 46 older adults were provided with a range of sensors deployed in their home for 2 years and compared with a non-equipped group of 67 older adults. Assessment of participants' functional, physical and cognitive status, through standardized clinical measures, showed maintenance after 2 years for equipped participants' while the control group's status significantly decreased over time.

However, one drawback of these two studies is the silo aspect of AAL technology. Indeed, the PRISM system only provides services for communication and leisure, and Rantz and Tomita's platforms are focused on activity analysis. Moreover, the efficacy of the technology was only estimated by the improvement of older adults' status, without involving caregivers' well-being. Recently, Dupuy and colleagues (2017) published results from a 6-month field study inves- tigating the effects of a multi-domain AAL platform toward frail older adults and their caregivers. In their study, 16 older care-receivers and their caregivers using the platform were compared to control dyads without equipment, and their results suggested benefits of the platform to support older adults' independence and relieve caregiver burden. However, these results would be strengthened by the evaluation of longerterm effects of the platform and prevent possible novelty effects. Indeed, alongside both efforts of study-design strength (control group, measurement reliability, etc.) and ecological validity, it is critical to investigate the durability of the clinical effects of AAL. Durability, also called maintenance effect, refers to the length of time therapeutic effects are maintained (Ardoin, 2006). It is an expected outcome measurement when investigating the effect of clinical intervention and is typically assessed with a short- to mild or longterm longitudinal follow-ups, to distinguish the near and far effects of the intervention. When both near and far effects are observed, this supports the durability of the intervention effect. Contrariwise, when near effects appeared but far effects fail to be observed, intervention durability is raised into question. Such findings obviously lead to moderate the extent of intervention effect or even to evoke possible novelty effects such as the well-known technology honeymoon effect in the field of adoption of technology innovations (Wells et al., 2010).

\section{Aim and research questions}

In this context, the aims of the present paper were the following:

1. Evaluate over a long-term deployment the efficacy of an AAL platform in terms of support for older adults' everyday functioning and caregiver burden according to a real-life setting;

2. Compare short-term effects to longer-term use benefits to identify possible novelty artefacts;

3. Leverage observed results to propose some research avenues for the design and evaluation of AAL technologies for the older adult and their caregivers.

\section{Methods}

We will present here the details of our experimental validation protocol: we will first describe our participant sample, then the AAL platform studied, named HomeAssist, the measures we used, and our experimental design.

\section{Participants}

As previously mentioned, this study included both older adults and their caregivers in an ecological situation, that is, older adults living in their homes. Therefore, we recruited dyads of one older adult and his/her professional caregiver, thanks to a collaboration with public home 


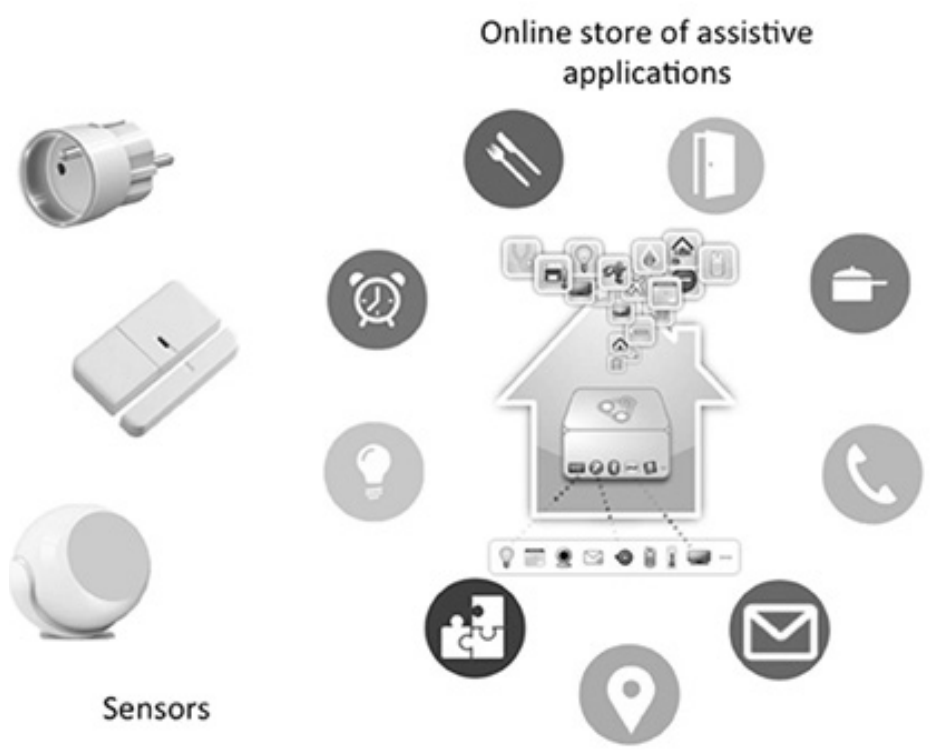

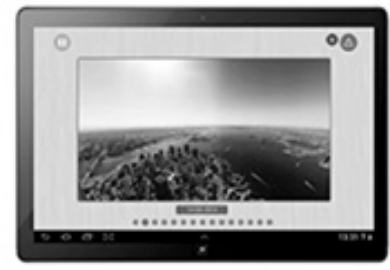

Main tablet: notification for ADL \& safety

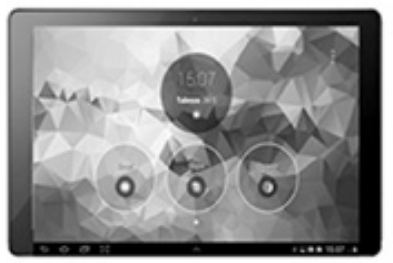

Secondary tablet: social activities

Figure 1. Content of the HomeAssist platform: A set of sensors, an online catalog of assistive applications and two touchscreen tablets

cal validation (i.e., applied to everyday life rather than laboratory setting; Schumkler, 2001) of HomeAssist efficacy.

\section{HomeAssist}

The platform was constituted by a set of sensors and actuators, as well as two touchscreen tablets (Figure 1).

Precisely, three types of sensors were used in our platform: contact sensors, enabling to detect when a door or a drawer is opened or closed; electric meters, sensing electric consumption of electric appliances and enabling to remotely turn it on or off; and motion sensors, collecting timed information when motion is detected in their sensing range. An example of an installation in a house is provided in Annex 2. These sensors were chosen for being small, wireless, cheap and respecting users' privacy. Users were interacting with the platform via two touchscreen tablets, operating under Android OS, that we redesigned following guidelines for the older population (e.g., ISO/TR 22411; Fisk et al., 2009) and prior user testing (Consel et al., 2015; Caroux et al., 2017).

Assistive services were provided by a catalog of online assistive applications, where each application could be downloaded or deleted depending on the evolutive needs of the users. The catalog provided services in three domains of everyday life: activities of daily living, safety, and communication and leisure, described hereafter.

(1) Applications for everyday activities
Our platform had the capacity to verify the appropriate realization of 5 everyday activities: getting up, meal preparation, toileting, dressing and going to bed; thanks to the set of sensors which placement was based on individual routine and housing type (Caroux et al., 2014). Moreover, via an online calendar accessible to caregivers, the platform could remind users of appointments and events.

\section{(2) Applications for safety}

Thanks to the range of sensors, several applications for safety could be proposed: first, a light path to prevent falls during the night, which was triggered by an electric meter sensing when the user turns on his bedside lamp. Second, a sensor disposed of on the entrance door enabled to detect if the door was left open or unexpected wandering during night. Lastly, electric appliances such as the stove could be monitored and automatically switched off. In case of critical situations detected by the platform, a text message was sent to a caregiver.

\section{(3) Applications for social participation}

Users were provided with a dedicated tablet proposing a simplified mailing system, enabling users to send emails using voice recording and a speech synthesizer to read the messages out loud (Caroux et al., 2017). This tablet also proposed video telephoning, web search engines, gaming apps, and social media apps, depending on users' needs. Users could also be informed regarding social events happening in their town through a mailing list. 


\section{Control condition}

In order to provide a comparable condition, we provided the control group with fake paperbased sensors and tablet, located at the same place as actual devices would be. We made that choice in order to introduce as well a new and unfamiliar material in participants' homes, and therefore reduce novelty effect; while not spending money in buying material that would not be used. In this case, participants noticed that something was new in their home, and some participants even asked if the sensor was recording anything. Beside, paper-based sensors, considered as technology probes, have been used in participatory design studies involving older adults and showed that it can successfully inspire older adults and provide guidelines for the design of future technologies (Plaisant et al., 2004).

\section{Experimental design}

The study was approved by the French Committee for Person Protection (CPP), the National Commission of Informatics and Liberty (CNIL) and the Ethics committee of the National Institute of Informatics and Mathematics (COERLE), as protecting participants and data accordingly.

The study involved 32 older adults and their caregivers over a period of 9 months. Sixteen participants benefited from HomeAssist during this time, while the other half received a fake, paperbased version of the platform disposed of in the place of actual sensors and tablets. The positioning of the set of sensors (either the fake or actual ones) was based on older adult's routine and housing type, analyzed by an occupational therapist during a previous visit.

For the equipped group, the deployment was performed by a home automation specialist, and then older adults and their caregivers benefitted from 4 training sessions to learn how to use the different services proposed by the platform. Users were also provided with a written manual and had access to a hotline in case of questions or difficulties. Lessons learned from this deployment process can be found in Dupuy \& Sauzéon, (2018).

Once these training sessions were over, we started the experimental trial and the following measures of interest were administrated to our two groups of participants, with three points of measure: at baseline $\left(\mathrm{t}_{0}\right)$, six months later $\left(\mathrm{t}_{6}\right)$ and after nine months $\left(t_{9}\right)$.

\section{Measures}

Both our equipped and control groups underwent assessments to evaluate older adults' functional status, and their caregivers were evaluated regarding their perceived burden in assisting their recipient. To do so, we used the following neuropsychological tools.

\section{Older adults' everyday functioning}

Older adults' functional status was quantified by two scales: first, the IADL scale (Lawton \& Brody, 1969), answered by the participant. This scale is constituted by 24 items screening for difficulties regarding several $A D L$, based on a 5-point Likert scale, a higher score indicating a greater range of difficulties. Additionally, older adult's functional status was hetero-evaluated by their caregiver (thanks to the French IHVA scale (Inventaire des Habiletés pour la Vie en Appartement; Corbeil et al., 2009; see Bruininks, 1984 for the original English version). Indeed, since we had the opportunity to meet with caregivers, we chose to perform a hetero-evaluation of older adults' functional status, as frail older adults are shown to be sometimes not accurate in self-evaluating their everyday difficulties (e.g., Kempen et al., 1996). This scale is composed of 12 dimensions, each of them comprising 10 items rating from 0 to 3 the ability to perform autonomously several ADL. Therefore, this scale induces a total score of 360, higher scores indicating a higher functional status reported by the caregiver.

\section{Caregiver burden}

Caregivers underwent two scales: first, the Maslach Burnout Inventory (MBI, Maslach et al., 1997), evaluating three aspects of subjective burden: emotional exhaustion, depersonalization, and personal accomplishment; over a 7-point Likert scale ranging from 0: "Never" to 6: "Always". We computerized a total score following Aloha and colleagues' (2014) formula: [0.4*exhaustion $+0.3 *$ depersonalization $+0.3 *(48$ - personal accomplishment)], inducing a higher total score in case of higher subjective professional burden. We also administrated an adaptation of Lawton's IADL scale, which we named Support for IADL scale, and focusing on the objective burden, i.e., the burden of assisting older adults performing their ADL. For example, an item was: "For you, the support that you provide for eating is: 0: "Very easy" - 6: "Very hard". Therefore, this scale for IADL support induces a global score of 144 , a higher score indicating more difficulties in assisting the older adult.

\section{Statistical analyses}

To evaluate the impact of HomeAssist on its users, we used mixed ANOVA with the following statistical design: Time as an intra-individual independent factor with three modalities $\left(t_{0} v s\right.$. $\mathrm{t}_{6}$ vs. $\left.\mathrm{t}_{9}\right)$; and Group as an inter-individual independent factor with two modalities (equipped vs. control groups). Knowing that our sample was relatively small, we performed statistical verification for using parametric tests: for within-subject data, Mauchly's test of sphericity was performed. 


\section{Field study of an assisted living platform for older adults}
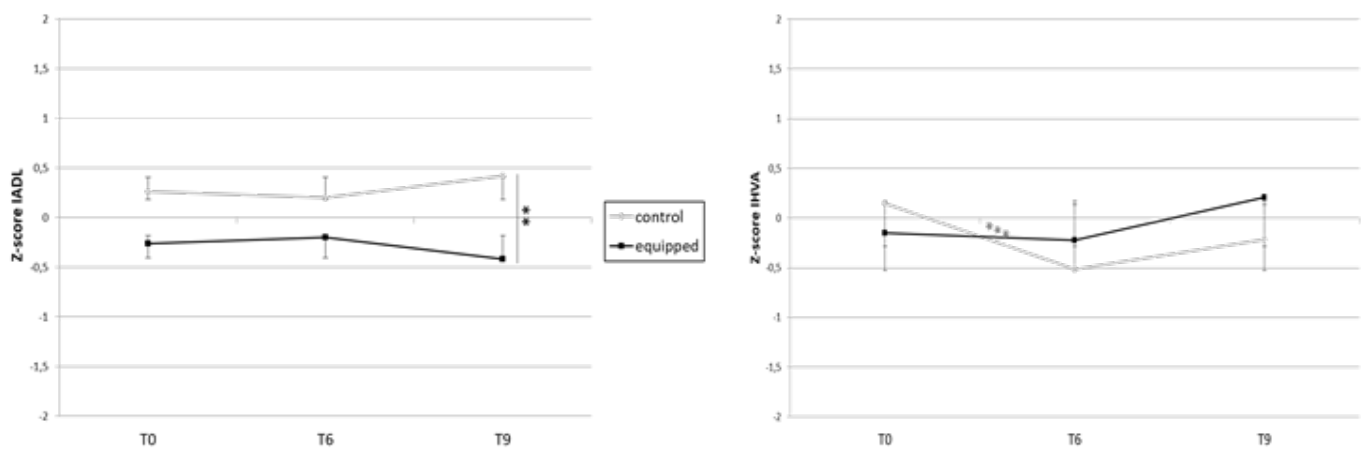

Figure 2. Evolution of older adults' autonomy for equipped and control group. Left: Self-reported measure of everyday difficulties (IADL scale). Right: Caregiver-reported measure of older adult's autonomy (IHVA scale). Statistics: ${ }^{* *}: p>0.01 ;{ }^{* * *}: p>0.001$

When the null hypothesis was significant (i.e., sphericity assumption was violated), corrections using Huynh-Feldt Epsilon $(\varepsilon)$ were reported instead of $\mathrm{p}$ significance (Huynh-Feldt Epsilons are known to be best suited for small samples; Huynh \& Feldt, 1976). For inter-subject data, Levene's test of homogeneity was performed, and when it came out significant, we reported Kruskal-Wallis test instead. Our four dependent measures were the total scores of the previously mentioned tests: the IADL scale, the IHVA scale, the MBI and the Support for IADL scale; and their Z-scores were computed in order to plot more comparable data. In case of statistical significance, posthoc tests were performed to compare between the three modalities of Time; and Student t-tests were used to compare between our two groups of participants. All statistical analyses were carried out with SAS SPSS Statistics 22.

\section{Results \\ Benefits for older adults' autonomy}

The evolution of older adults' autonomy for our two groups of participants is plotted in Figure 2.

Regarding older adults' perception of their autonomy to perform ADL (i.e., scores of the IADL scale, Figure 2 left), results of the mixed ANO-
VA did not show any significant global effect of Time ( $p>.900)$, Group ( $p>.06)$, nor interaction Time*Group $(\varepsilon>.200)$. Nonetheless, when looking into differences between groups, results suggest that after 9 months, equipped participants perceived themselves as more autonomous than the control group $(p=.015 ; t=2.589)$.

Regarding caregivers' evaluation of older adults' functional status (i.e., the IHVA score, Figure 2 right), global effect of Time was not significant ( $p$ $>$.900) nor effect of Group ( $p>$.600). However, a significant interaction effect Time*Group was obtained $\left[\mathrm{F}(2,29)=11.31 ; \varepsilon<0.001 ; \eta^{2}=0.27\right]$. Precisely, comparisons showed that according to caregivers, the equipped group's autonomy remains constant from to to t9; whereas the score decreased significantly between $t 0$ and $t 6$ for the control group $(\mathrm{p}<0.001)$.

\section{Benefits for caregiver burden}

Evolution over time of caregiver burden for equipped and control groups is illustratedin Figure 3.

First, regarding perceived professional burden (Figure 3 left), no significant effects were found, showing no differences between groups and overtime of the $\mathrm{MBI}$ score.
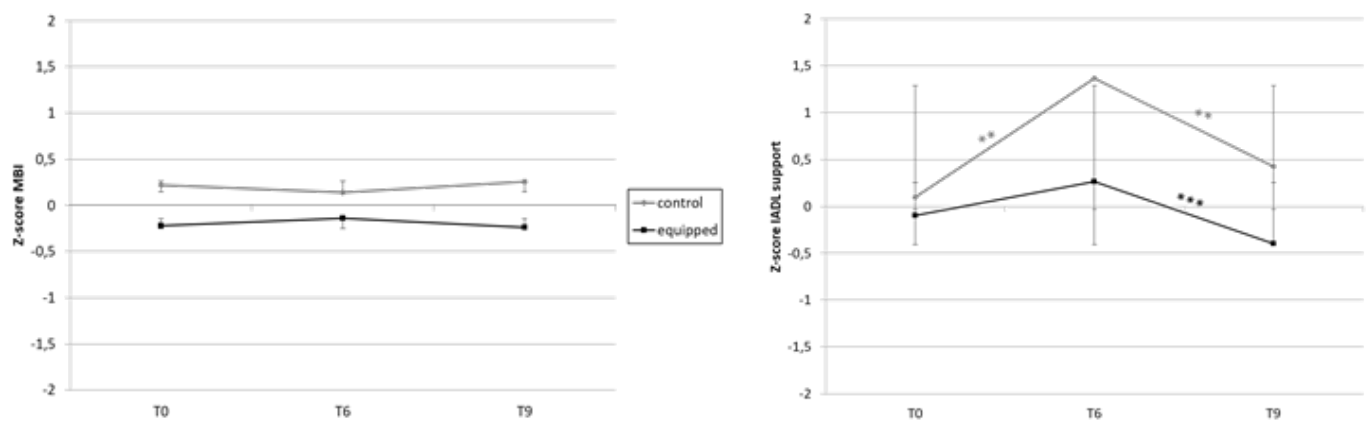

Figure 3. Evolution of caregiver burden for equipped and control group. Left: Professional burden (MBI scale). Right: Burden in assisting the older adult (Support for IADL scale). Statistics: ${ }^{* *}: p>0.01$; ${ }^{* * *}$ : $p>0.001$ 
Regarding the Support for IADL score (Figure 3 right), assessing caregivers' perceived difficulties to support their recipient, results of the mixed ANOVA show a significant interaction effect Time*Group $\left[F(2,29)=4.31 ; \varepsilon=0.039 ; \eta^{2}\right.$ $=0.13]$. Post-hoc analyses showed that for the equipped group, caregiver burden remained stable between $t_{0}$ and $t_{6}$, and significantly decreased from $t_{6}$ to $t_{9}(p=0.001)$. By comparison, for the control group, the burden significantly increased from $t_{0}$ to $t_{6}(p=0.001)$ and then significantly decreased from $t_{6}$ to $t_{9}(p=0.001)$. Additionally, significant group differences were obtained at $t_{6}$ $[\mathrm{t}(30)=2.27 ; \mathrm{p}=0.03]$ and $\mathrm{tat}_{9}[\mathrm{t}(30)=2.39 ; \mathrm{p}=$ $0.02]$, with, in both case, caregivers in the control group reporting more difficulties assisting their care-receivers than in the equipped group.

\section{Discussion}

In this study, we were interested in evaluating the impact of our platform HomeAssist to support older adults' autonomy and relieve caregiver burden. To do so, we compared 16 older Fls using our platform at home for nine months, with a control group receiving a fake version of the technology.

Regarding benefits for everyday functioning, we observed that older adults' self-perceived difficulties varied little over time and among the group, suggesting only a slight impact of HomeAssist in Fls' self-perception of everyday functioning. Interestingly, HomeAssist benefits are tangible on self-perception scores only after 9 months. When interrogating their caregivers, results showed maintenance of equipped $\mathrm{FIs}^{\prime}$ autonomy after nine months of use, whereas reported everyday functioning decreased in the control group. These results have several implications: first, it highlights the well-known discrepancies occurring in aging between self-evaluation and heteroevaluation of everyday functioning, suggesting difficulties for older adults to accurately estimate their difficulties, as found in other studies (Kempen et al., 1996; Gold, 2012). When evaluating technology efficacy, it is therefore recommended not to solely rely on older adults' self-evaluation, but rather include objective measures (e.g., measures of usage, objective scales of everyday functioning) or hetero-evaluation by family or caregivers. In addition, according to caregivers, our results show a significant increase of users everyday functioning compared to the control group, occurring during the last three months of use. Indeed, these results suggest that the use of HomeAssist was associated with the maintenance of everyday functioning during the six first months of use, followed by increased everyday functioning at nine months. Hence, HomeAssist benefits appear to be more of a long-term nature. These time-related gradual benefits can be interpreted as mirroring progressive Fls' appro- priation of HomeAssist as assistive devices. In a previous study on HomeAssist usability (Consel et al., 2015), maximal effectiveness performance (assessed by interaction accuracy) was reached after 4.5 months of using our platform, while maximal usability efficiency (interaction time) was reached at six months of use. Taken together, these two observations were seen as automation of the process of answering assistive notifications, suggesting effective learning of the platform over time. Probably, automatized or proficient interactions with HomeAssist are required to bring actual benefits, i.e. improving everyday activities. Such interpretation would deserve further investigations to compare observed benefits according to the user's proficiency with HomeAssist. Overall, results suggest positive benefits of HomeAssist to support Fls' autonomy and highlights the importance of long-term studies to evaluate technology's efficacy, particularly when targeted users are not technophile and technology appropriation might delay its benefits.

Regarding the caregiver burden, we observed that HomeAssist does not influence caregiver's professional burden over time. This result can be seen positively as it suggests that the deployment of HomeAssist in older adults' homes does not induce a supplementary burden on caregivers' activity. Indeed, some studies highlighted that this concern is often raised as a limitation to technology adoption by professional caregivers themselves (Schulz et al., 2015), and by older adults who are worried about adding burden in their caregivers' life (e.g., Peek et al., 2014, Lorenzen-Huber et al., 2011). Interestingly, our results indicated that caregivers' perceived burden to support older adults is positively impacted by HomeAssist, as in the equipped group, caregivers' difficulties remain constant during the first six months of use and decrease over the last three months (while they increased for control group), suggesting that they become more comfortable in their role over time.

It is noteworthy that surprisingly, caregivers' perceived burden to support older adults is diminished at nine months' follow-up irrespective of group condition (albeit group differences remained significant). A possible explanation could be the shorter time interval between $t_{6}$ and $t_{9}$ (compared to that of $t_{0}$ and $t_{6}$ ), eliciting biased responses, as respondents might modulate their answers according to memories of the previous assessment.

Taken together, our results suggest the benefits induced by HomeAssist to support older adults' autonomy and relieve caregiver burden. On these two dimensions, we observed a stronger effect occurring during the last three months of use compared to the effects observed after six 
months, dispelling possible novelty effects.

\section{Limitations}

Despite our positive results, some limitations can be raised. First, the dropout rate occurring along with our study, due to real-life and long-term settings hazards, might imply that our final sample includes only participants who had positive experiences with HomeAssist, biasing our results.

Second, our final sample might seem relatively small, questioning the statistical power of our results. However, one can hope stronger results with a bigger sample, as even when statistical significance was not fully reached, we noted tendential positive results.

Third, one can argue that our control condition might be biased as it is a passive condition, i.e., does not provide any similar content to the equipped group. Nonetheless, by deploying fake sensors in the home of our control participants, we still added some new and unfamiliar objects to older adults' everyday life, therefore reducing novelty effects. Additionally, it would be interesting to compare two technological supports providing different functionalities, in order to highlight the benefits of each functionality in technology acceptance.

Fourth, a longer period interval such as 12 or 18 months rather than 9 months would provide more insights regarding near and far effects. However, our purpose was basically to assess the technology honeymoon effect. To the best of our knowledge, such purpose in the AAL domain has not been investigated.

Finally, we could have added informal caregivers (e.g., family, friends) in our assessments, to investigate additional possible benefits regarding the reduction of their caregiving burden. For example, the widely used psychosocial scale Zarit (Zarit, Reever \& Bach-Peterson, 1980) could be administrated in a future study.

Despite these limitations, our study is a necessary first step toward large-scale field study to demonstrate the efficacy of the AAL platform. Our results gave encouraging results regarding near and far HomeAssist's efficacy to support older adults' autonomy and well-being, as well as relieving caregiver burden.

\section{Implications for research and design}

The present study suggests several implications for the future of research and design in the context of technologies for aging.

First, it highlights the benefits of designing longterm and ecological studies to evaluate the im- pact of AAL technologies. Indeed, as well as inducing more generalizable and ecological results, it enables us to face real-life settings challenges of including new technology in older adults' life, that would have remained unseen between the walls of a laboratory setting.

Second, when evaluating the efficacy of the technology, our results suggest the added value of involving caregivers in the process, both to collect a hetero-evaluation of technology's impact over older adults' everyday life, and because their opinion might influence older adults' opinion toward the technology and affect its adoption.

Third, this study showed the added value of having a control condition in an evaluation methodology, particularly with an aging population. Indeed, it showed that older adults' status is evolving even without adding technological support, and the comparison between the experimental and the control condition enables to bring forward the specific benefits of the technology in older adults' everyday life.

Fourth, we recommend the application of User-Centered Design methodologies to develop technologies for aging. Indeed, in our case, following users' needs (Dupuy, Sauzéon \& Consel, 2015) enabled to adapt HomeAssist functionalities, design, and deployment methodology. Notably, the necessity of personalization, a learning phase provided on a step-by-step basis and the involvement of caregivers in all the phases of the study were major takeaway ideas (Dupuy, Consel \& Sauzéon, 2016; Dupuy \& Sauzéon, 2018).

Fifth, regarding commercialization objectives. Our research methodology was regulated by committees in order to protect participants and data. However, as suggested by Torous \& Haim (2018) in their recent article, when it comes to commercialization of health apps, choices have to be made. Notably, a balance has to be found between Big Data vs. Privacy, data sharing vs. proprietary tools or free-market vs. medical regulation.

Finally, our platform shows the benefits of developing multi-domain and adaptive platforms, adjusting to the various needs of Fls. Indeed, it enables to give a unified interface to the user, therefore reducing the learning cost, to propose individualized support depending on the various and evolving needs of older adults, and to aggregate the collected data to create a more global picture of older adult's status.

\section{Conclusion}

To summarize, this study proposed to evaluate the durable benefits of AAL technology in real- 
setting by deploying a multi-domain assistive platform in the house of frail older adults, and compare with a control group. Our results highlighted the positive influence of the platform to maintain and improve older adults' everyday functioning while reducing caregiver burden.
Additionally, such results appear to be not elicited by the technology's honeymoon effects. Finally, this study enabled us to raise some recommendations for research and design of AAL in the context of aging, providing fruitful avenues for the future of technologies for aging.

\section{References}

Ardoin, N. M. 2006. Toward an interdisciplinary understanding of place: lessons for environmental education. Canadian Journal of Environmental Education 11:112-126.

Avila-Funes, J. A., Helmer, C., Amieva, H., BarbergerGateau, P., Goff, M. L., Ritchie, K., \& Dartigues, J. F. (2008). Frailty among community-dwelling elderly people in France: the three-city study. The Journals of Gerontology Series A: Biological Sciences and Medical Sciences, 63(10), 1089-1096.

Berkowsky, R. W., Sharit, J., \& Czaja, S. J. (2018). Factors predicting decisions about technology adoption among older adults. Innovation in Aging, 1(3), 1-12.

Bruininks, R. H. (1984). Scales of Independent Behavior: Psycho-educational Battery. DLM.

Caroux, L., Consel, C., Dupuy, L., and Sauzéon, H. (2014). "Verification of daily activities of older adults: a simple, non-intrusive, low-cost approach," in Proceedings of the 16th International ACM SIGACCESS Conference on Computers \& Accessibility (Rochester, NY), 43-50.

Caroux, L., Consel, C., Dupuy, L., and Sauzéon, H. (2017). "Designing an accessible and engaging email application for aging in place," in The 14th of IEEE Ubiquitous Intelligence and Computing (UIC 2017) (San Francisco, CA).

Clegg, A., Young, J., Iliffe, S., Rikkert, M. O., \& Rockwood, K. (2013). Frailty in elderly people. The lancet, 381(9868), 752-762.

Collard, R. M., Boter, H., Schoevers, R. A., \& Voshaar, R. C. O. (2012). Prevalence of frailty in community-dwelling older persons: a systematic review. Journal of the American Geriatrics Society, 60(8), 1487-1492.

Corbeil, R., Marcotte, A., \& Trépanier, C. (2009). Inventaire des habiletés pour la vie en appartement. Québec: Groupe de recherche et d'étude en déficience du développement.

Czaja, S. J., Boot, W. R., Charness, N., Rogers, W. A., \& Sharit, J. (2017). Improving social support for older adults through technology: findings from the PRISM randomized controlled trial. The Gerontologist, 58(3), 467-477.

Dupuy, L., \& Sauzéon, H. (2018). Ambient assisted living platforms for aging in place: lessons learned from a field study. Gerontechnology, 17(s), 109.

Dupuy, L., Froger, C., Consel, C., \& Sauzéon, H. (2017). Everyday Functioning Benefits from an Assisted Living Platform amongst Frail Older Adults and Their Caregivers. Frontiers in aging neuroscience, 9, 302.

Dupuy, L., Consel, C., \& Sauzeon, H. (2016). Self-determination-based design to achieve acceptance of assisted living technologies for older adults. Computers in Human Behavior, 65, 508-521.
Dupuy, L., Sauzéon, H., and Consel, C. (2015). "Perceived Needs for Assistive Technologies in older adults and their caregivers," in WomENcourage 15'.

Durick, J., Robertson, T., Brereton, M., Vetere, F., \& Nansen, B. (2013, November). Dispelling ageing myths in technology design. In Proceedings of the 25th Australian Computer-Human Interaction Conference: Augmentation, Application, Innovation, Collaboration (pp. 467-476). ACM.

Folstein, M. F., Folstein, S. E., and McHugh, P. R. (1975). Mini-mental state. Journal of Psychiatric Research 12, 189-198.

Fried, L. P., Tangen, C. M., Walston, J., Newman, A. B., Hirsch, C., Gottdiener, J., et al. (2001). Frailty in older adults evidence for a phenotype. The Journals of Gerontology Series A: Biological Sciences and Medical Sciences,56, M146-M157.

Gold, D. A. (2012). An examination of instrumental activities of daily living assessment in older adults and mild cognitive impairment. Journal of clinical and experimental neuropsychology, 34(1), 11-34.

Guigoz, Y, and Vellas, B. (1999) "The Mini Nutritional Assessment (MNA) for grading the nutritional state of elderly patients: presentation of the MNA, history and validation," in Mini Nutritional Assessment (MNA): Research and Practice in the Elderly, Nestle Nutrition Workshop Series Clinical \& Performance Programme, Vol. 1, eds B. Vellas, Ph. J. Garry, and Y. Guigoz (Basel: Nestec Ltd.; Vevey/S. Karger AG), 3-12.

Guralnik, J. M., Ferrucci, L., Pieper, C. F., Leveille, S. G., Markides, K. S., Ostir, G. V., et al. (2000). Lower extremity function and subsequent disability: consistency across studies, predictive models, and value of gait speed alone compared with the short physical performance battery. The Journals of Gerontology Series A: Biological Sciences and Medical Sciences, 55, 221-231.

Huynh, H., \& Feldt, L. S. (1976). "Estimation of the Box correction for degrees of freedom from sample data in randomised block and split-plot designs." Journal of Educational Statistics, 1, 69-82.

Kalafat, M., Hugonot-Diener, L., \& Poitrenaud, J. (2003). Standardisation et étalonnage français du "Mini Mental State"(MMS) version GRECO. Revue de neuropsychologie, 13(2), 209-236.

Kempen, G. I., Steverink, N., Ormel, J., \& Deeg, D. J. (1996). The assessment of ADL among frail elderly in an interview survey: self-report versus performance-based tests and determinants of discrepancies. The Journals of Gerontology Series B: Psychological Sciences and Social Sciences, 51(5), P254-P260.

Lawton, M.P., \& Brody, E.M. (1969). Assessment of older people: Self-maintaining and instrumental activities 
of daily living. Gerontologist, 9(3 Part 1):179-186.

Lee, M. L., \& Dey, A. K. (2014, April). Real-time feedback for improving medication taking. In Proceedings of the SIGCHI Conference on Human Factors in Computing Systems (pp. 2259-2268). ACM.

Lorenzen-Huber, L., Boutain, M., Camp, L. J., Shankar, K., \& Connelly, K. H. (2011). Privacy, technology, and aging: a proposed framework. Ageing International, 36(2), 232-252.

Lindley, S. E. (2012). Shades of lightweight: supporting cross-generational communication through home messaging. Universal Access in the Information Society, 11(1), 31-43.

Liu, L., Stroulia, E., Nikolaidis, I., Miguel-Cruz, A., \& Rincon, A. R. (2016). Smart homes and home health monitoring technologies for older adults: A systematic review. International journal of medical informatics, 91, 44-59.

Maslach, C., Jackson, S. E., \& Leiter, M. P. (1997). Maslach burnout inventory. Evaluating stress: A book of resources, 3, 191-218.

McFall, S., \& Miller, B. H. (1992). Caregiver burden and nursing home admission of frail elderly persons. Journal of Gerontology, 47(2), S73-S79.

Mihailidis, A., Boger, J. N., Craig, T., \& Hoey, J. (2008). The $\mathrm{COACH}$ prompting system to assist older adults with dementia through handwashing: An efficacy study. BMC Geriatrics, 8(1), 28.

Montgomery, R. J., Gonyea, J. G., \& Hooyman, N. R. (1985). Caregiving and the experience of subjective and objective burden. Family relations, 19-26.

Morley, J. E., Vellas, B., Van Kan, G. A., Anker, S. D., Bauer, J. M., Bernabei, R., \& Fried, L. P. (2013). Frailty consensus: a call to action. Journal of the American Medical Directors Association, 14(6), 392-397.

Morrow, D. G., \& Rogers, W. A. (2008). Environmental support: An integrative framework. Human Factors, 50(4), 589-613.

Neves, B. B., Franz, R. L., Munteanu, C., Baecker, R., \& Ngo, M. (2015, April). My Hand Doesn't Listen to Me!: Adoption and Evaluation of a Communication Technology for the 'Oldest Old'. In Proceedings of the 33rd Annual ACM Conference on Human Factors in Computing Systems (pp. 1593-1602). ACM.

Peek, S. T., Wouters, E. J., van Hoof, J., Luijkx, K. G., Boeije, H. R., \& Vrijhoef, H. J. (2014). Factors influencing acceptance of technology for aging in place: a systematic review. International journal of medical informatics, 83(4), 235-248.

Plaisant, C., Clamage, A., Hutchinson, H. B., Bederson, B. B., \& Druin, A. (2006). Shared family calendars: Promoting symmetry and accessibility. ACM Transactions on Computer-Human Interaction (TOCHI), 13(3), 313-346.

Queirós, A., Silva, A., Alvarelhão, J., Rocha, N. P., and Teixeira, A. Usability, accessibility and ambient-assisted living: a systematic literature review. Universal Access in the Information Society, 14, 1 (2015), 57-66.
Rantz, M., Phillips, L. J., Galambos, C., Lane, K., Alexander, G. L., Despins, L., \& Craver, A. (2017). Randomized trial of intelligent sensor system for early illness alerts in senior housing. Journal of the American Medical Directors Association, 18(10), 860-870.

Rashidi, P., \& Cook, D. J. (2009). Keeping the resident in the loop: Adapting the smart home to the user. IEEE Transactions on systems, man, and cyberneticspart A: systems and humans, 39(5), 949-959.

Reeder, B., Meyer, E., Lazar, A., Chaudhuri, S., Thompson, H. J., and Demiris, G. Framing the evidence for health smart homes and home-based consumer health technologies as a public health intervention for independent aging: A systematic review. International Journal of Medical Informatics, 82, 7 (2013), 565-579.

Rocha, A., Martins, A., Junior, J. C. F., Boulos, M. N. K., Vicente, M. E., Feld, R., \& Sdogati, C. (2013). Innovations in health care services: The CAALYX system. International journal of medical informatics, 82(11), e307-e320.

Schulz, R., Beach, S. R., Matthews, J. T., Courtney, K., De Vito Dabbs, A., \& Mecca, L. P. (2015). Caregivers' willingness to pay for technologies to support caregiving. The Gerontologist, 56(5), 817-829.

Slim, K., Nini, E., Forestier, D., Kwiatkowski, F., Panis, Y., \& Chipponi, J. (2003). Methodological index for non-randomized studies (MINORS): development and validation of a new instrument. ANZ journal of surgery, 73(9), 712-716.

Schmuckler, M. A. (2001). What is ecological validity? A dimensional analysis. Infancy, 2(4), 419-436.

Sposaro, F., \& Tyson, G. (2009, September). iFall: an Android application for fall monitoring and response. In Engineering in Medicine and Biology Society, 2009. EMBC 2009. Annual International Conference of the IEEE (pp. 6119-6122). IEEE.

Theou, O., Stathokostas, L., Roland, K. P., Jakobi, J. M., Patterson, C., Vandervoort, A. A., \& Jones, G. R. (2011). The effectiveness of exercise interventions for the management of frailty: a systematic review. Journal of aging research, 2011.

Torous, J., \& Haim, A. (2018). Dichotomies in the Development and Implementation of Digital Mental Health Tools. Psychiatric Services, 69(12), 1204-1206.

Ware Jr, J. E., \& Sherbourne, C. D. (1992). The MOS 36-item short-form health survey (SF-36): I. Conceptual framework and item selection. Medical care, 473-483.

Wells, J. D., Campbell, D. E., Valacich, J. S., \& Featherman, M. (2010). The effect of perceived novelty on the adoption of information technology innovations: a risk/reward perspective. Decision Sciences, 41(4), 813-843.

Zarit, S. H., Reever, K. E., \& Bach-Peterson, J. (1980). Relatives of the impaired elderly: correlates of feelings of burden. The gerontologist, 20(6), 649-655. 


\section{Field study of an assisted living platform for older adults}

ApPendix I - Detailed means and Standard deviations of our fOUR MEASURes of INTERest: IADL, IHVA, MBI AND IADL SUPPORT

\begin{tabular}{|c|c|c|c|c|c|c|c|c|c|c|c|c|}
\hline & \multicolumn{3}{|c|}{$\begin{array}{l}\text { IADL } \\
\text { M (SD) }\end{array}$} & \multicolumn{3}{|c|}{$\begin{array}{l}\text { IHVA } \\
\text { M (SD) }\end{array}$} & \multicolumn{3}{|c|}{$\begin{array}{c}\text { MBI } \\
\text { M (SD) }\end{array}$} & \multicolumn{3}{|c|}{$\begin{array}{c}\text { IADL Support } \\
\text { M (SD) }\end{array}$} \\
\hline & T0 & T6 & T9 & T0 & T6 & T9 & T0 & T6 & T9 & T0 & T6 & T9 \\
\hline Equipped & $\begin{array}{l}-0.26 \\
(0.75)\end{array}$ & $\begin{array}{l}-0.27 \\
(0.81)\end{array}$ & $\begin{array}{l}-0.38 \\
(0.74)\end{array}$ & $\begin{array}{l}-0.15 \\
(0.86)\end{array}$ & $\begin{array}{l}-0.22 \\
(0.82)\end{array}$ & $\begin{array}{c}0.20 \\
(0.86)\end{array}$ & $\begin{array}{l}-0.22 \\
(0.68)\end{array}$ & $\begin{array}{l}-0.14 \\
(0.77)\end{array}$ & $\begin{array}{l}-0.24 \\
(0.91)\end{array}$ & $\begin{array}{l}-0.09 \\
(0.94)\end{array}$ & $\begin{array}{c}0.26 \\
(0.97)\end{array}$ & $\begin{array}{l}-0.40 \\
(0.62\end{array}$ \\
\hline Control & $\begin{array}{c}0.26 \\
(1.15)\end{array}$ & $\begin{array}{c}0.06 \\
(1.12)\end{array}$ & $\begin{array}{c}0.40 \\
(1.11)\end{array}$ & $\begin{array}{c}0.15 \\
(1.13)\end{array}$ & $\begin{array}{l}-0.51 \\
(1.21)\end{array}$ & $\begin{array}{l}-0.22 \\
(1.12)\end{array}$ & $\begin{array}{c}0.22 \\
(1.22)\end{array}$ & $\begin{array}{c}0.14 \\
(1.19)\end{array}$ & $\begin{array}{c}0.25 \\
(1.06)\end{array}$ & $\begin{array}{c}0.09 \\
(1.08)\end{array}$ & $\begin{array}{c}1.36 \\
(1.67)\end{array}$ & $\begin{array}{r}0.42 \\
(1.16\end{array}$ \\
\hline
\end{tabular}

Appendix II - Example of HomeAssist deployment in a Patricipant's house. Types of Sensors and ACTUATORS ARE PRESENTED WITH DIFFERENT COLORS

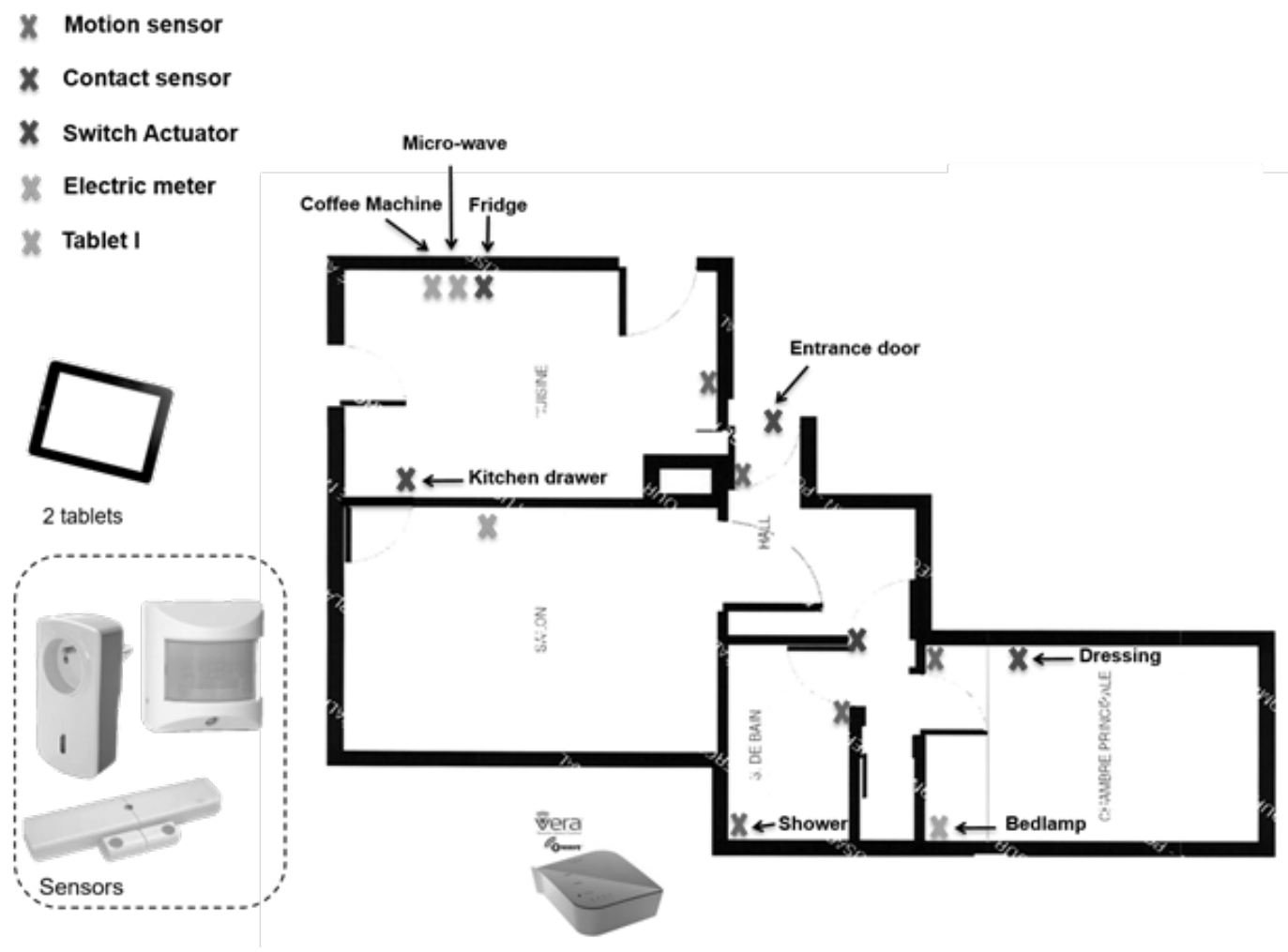

